\title{
Recent Results on Hadron Spectroscopy at COMPASS
}

\author{
Reiner Geyer*t \\ on the behalf of the COMPASS collaboration \\ Faculty of Physics, Ludwig-Maximilians-University Munich, \\ Schellingstrasse 4, 80799 Munich \\ E-mail: Reiner.Geyerecern.ch
}

COMPASS is a multi-purpose fixed target experiment at CERN's Super Proton Synchrotron, dedicated to the study of the structure of the nucleon and the spectroscopy of hadrons. The large acceptance and high resolution two stage spectrometer takes advantage of the availability of a variety of high intensity beams (muons and hadrons) with momenta up to $300 \mathrm{GeV} / \mathrm{c}$. During two weeks in 2004 and the years 2008 to 2009, COMPASS has focused on the search for exotic hadronic states in central production and diffractive projectile excitation. $190 \mathrm{GeV} / \mathrm{c}$ hadron beams (mainly $p$ and $\pi^{-}$) were used on different target materials $\left(\mathrm{LH}_{2}, \mathrm{Ni}\right.$ and $\mathrm{Pb}$ ). Mesonic resonances with masses up to about $2.5 \mathrm{GeV} / \mathrm{c}^{2}$ are accessible in these production mechanisms at COMPASS. An overview on the results obtained from PWA and the status of the ongoing analysis will be given.

The Xth Nicola Cabibbo International Conference on Heavy Quarks and Leptons, October 11-15, 2010

Frascati (Rome) Italy

\footnotetext{
* Speaker.

${ }^{\dagger}$ Present address: Reiner Geyer, CERN, 1211 Geneve 23, Switzerland. Reiner.Geyer@ cern.ch.
} 


\section{Introduction}

The COMPASS (COmmon Muon and Proton Apparatus for Structure and Spectroscopy) experiment at CERN became operational in 2002. Until 2007, the main interest of the collaboration was focused on DIS measurements with $160 \mathrm{GeV} / c \mu^{+}$-beams on polarized solid targets in order to study the spin structure of the nucleon. Longitudinally and transversely polarized Deuterons $\left({ }^{6} \mathrm{LiD}, 2002-2006\right)$ and Protons $\left(\mathrm{NH}_{3}, 2007\right)$ were used as target materials. A variety of results has been meanwhile obtained covering subjects like the polarized structure function $g_{1}$ and its first

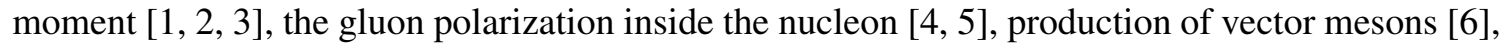
transverse spin effects [ $[\mathbb{Z}, \mathbb{Q}, \mathbb{Q}, \mathbf{Q}]$ or polarization of hyperons. Only a few days of effective data taking was dedicated in 2004 for hadron beams, when a short pilot run was carried out with $190 \mathrm{GeV} / \mathrm{c}$ negative pions on nuclear targets. Despite the shortness of this run, COMPASS collected a data sample with sufficiently large statistics to be competitive with earlier experiments. First results were obtained in the diffractive excitation of pions via the reaction $\pi^{-} N \rightarrow \pi^{-} \pi^{+} \pi^{-} N$. In 2008 and 2009, COMPASS dedicated its beam time completely for hadron spectroscopy in diffractive scattering and central production. $\pi, K$ and $p$ with $190 \mathrm{GeV} / c$ were used as beam particles on $\mathrm{LH}_{2}$ and nuclear targets. In the following, I will report on the meson spectroscopy results obtained from the 2004 pilot run in $\pi^{-} N \rightarrow \pi^{-} \pi^{+} \pi^{-} N$. I also will summarize the status and the perspectives of the ongoing analysis of 2008 and 2009 data and present first preliminary results.

\section{Meson Spectroscopy at COMPASS}

The simple $S U(3)_{\text {flavor }}$ constituent quark model provides an amazingly successful description of the spectrum of light mesons in terms of bound states of quarks $q$ with antiquarks $\bar{q}$ with the flavors $\mathrm{u}, \mathrm{d}$ or s. The states are grouped into multiplets with the quantum numbers $J^{P C}$, where $J$ is the total angular momentum, $P$ is the parity and $C$ is the charge conjugation parity. In addition the isospin $I$ and the $G$-parity are used to characterize these mesons. In the constituent quark model, the parity quantum numbers of mesons can be related to the orbital angular momentum $L$ and the total spin $S=0,1$ of the $q \bar{q}$-pairs: $(P)=-1^{L+1}, C=(-1)^{L+S}, G=(-1)^{I+L+S}$. Despite the success of the constituent quark model to describe most of the observed meson spectrum, color singlet states in QCD can not only be obtained by $q \bar{q}$ constituent quark pairs, but also in other combinations between quarks, antiquarks and gluons. Such states, like tetra-quark objects $(q \bar{q} q \bar{q})$, glueballs $(g g)$ or hybrids $(q \bar{q} g)$ have been predicted by theory but never unambiguously experimentally proven.

The lowest glueball states $0^{++}, 2^{++}$and $0^{-+}$are predicted by quenched lattice QCD at masses accessible at COMPASS, while glueballs with exotic quantum numbers $\left(0^{+-}, 2^{-+}, 1^{-+}\right)$should not appear below $3 \mathrm{GeV} / \mathrm{c}^{2}[\mathbb{[}]$ ]. Because the lowest glueballs have conventional quantum numbers with masses in a dense background of conventional $q \bar{q}$ states, it is difficult to distinguish them from conventional mesons. One of the significant properties of glueballs is their flavor blind decays, which might allow for their identifications. For the pure gluonic $0^{++}$state, the phase space corrected branching ratios are expected as follows: $\Gamma\left(G \rightarrow \pi \pi: K \bar{K}: \eta \eta: \eta \eta^{\prime}: \eta^{\prime} \eta^{\prime}\right)=3: 4: 1: 0: 1$. Non- $q \bar{q}$-configurations may mix with the ordinary mesonic states. Unfortunately, mixing can significantly alter the properties of the underlying states, which makes their interpretation more difficult. Experimentally the $f_{0}(1500)$, or, alternatively, the $f_{0}(1710)$ have been proposed as candidates 
for the scalar glueball, both states having considerable mixing also with the $f_{0}(1370)$. Other mixing schemes were also proposed [W]. It is essential to reconfirm and complete the experimental results of the resonances listed above. A good knowledge of their decay properties will be essential for a correct interpretation of their nature. Among others, such resonances $(\mathrm{X})$ can be studied in COMPASS by using the central production mechanism $p p \rightarrow p_{f} X p_{s}$ and observing their subsequent decays in $\pi \pi, K \bar{K}, \eta \eta, \eta \eta^{\prime}$ or $\eta^{\prime} \eta^{\prime}$. Nevertheless, due to the low CM energy, one of the experimental challenges of COMPASS will be the separation of central production from other production mechanisms like diffractive dissociation.

The observation of states with exotic quantum numbers, which are not accessible in the simple constituent quark model $\left(J^{P} C=0^{--}, 0^{+-}, 1^{-+}, \ldots\right)$ would give clear and direct evidence for the existence of quark-gluon configurations beyond the constituent quark model, as allowed by QCD. The hybrid with the lowest mass is predicted in some models to have the quantum numbers $J^{P C}=1^{-+}$and thus will not mix with ordinary mesons [ए2]. Its mass should be in the range between $1.3-2.2 \mathrm{GeV} / \mathrm{c}^{2}$, which lies within the COMPASS acceptance. Several experimental candidates were reported for such a state, the $\pi_{1}(1400)$ seen by E852 [[13], VES [ए4]] and Crystal

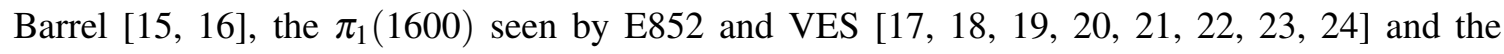
$\pi_{1}(2000)$ [22], 24]. The resonant nature of these states, however, is still heavily disputed in the community [144, [23]. One possibility at COMPASS to search for the production of hybrids is diffractive dissociation. Diffractive dissociation is a reaction of the type $a+b \rightarrow c+d$, where $a$ is the incoming beam particle, $b$ the target, $c$ is a via Reggeon or Pomeron exchange diffractively produced object and $d$ is the target recoil particle. The production kinematics is described by $t^{\prime}=|t|-\left|t_{\min }\right|$, where $t=\left(p_{a}-p_{c}\right)^{2}$ is the square of the four momentum transferred from the incoming beam to the outgoing system $c$ and $\left|t_{\min }\right|$ is the minimum value of $|t|$ which is required for the production of a mass $m_{c}$. COMPASS can cover $t$ values ranging from zero up to a few $(\mathrm{GeV} / \mathrm{c})^{2}$.

\section{Experimental Results}

During the Hadron Pilot Run in 2004 of only a few days of effective data taking, COMPASS collected a sufficiently large statistics in order to shed new light on the existence of the $\pi_{1}(1600)$ [25] . A $190 \mathrm{GeV} / \mathrm{c} \pi^{-}$-beam was used on a $3 \mathrm{~mm}$ lead target. The trigger was based on a multiplicity increase of charged particles after the target, which allowed selecting events of the type $\pi^{-} N \rightarrow \pi^{-} \pi^{+} \pi^{-} N$ (which corresponds to the diffractive process $a+b \rightarrow c+d$ with the subsequent decay $\left.c \rightarrow \pi^{-} \pi^{+} \pi^{-}\right)$. For the further analysis only events were used, where $t^{\prime}$ is in the range of $0.1(\mathrm{GeV} / \mathrm{c})^{2}<t^{\prime}<1(\mathrm{GeV} / \mathrm{c})^{2}$. Figure 1(a) shows the invariant mass spectrum between 0.5 and $2.5 \mathrm{GeV} / \mathrm{c}^{2}$ of the corresponding events. The well known resonances $a_{1}(1260), a_{2}(1320)$ and $\pi_{2}(1670)$ are clearly visible in the $\pi^{-} \pi^{+} \pi^{-}$invariant mass spectrum of 420000 events.

A partial wave analysis (PWA) of this data set was performed which is based on the phenomenological isobar model, in which all multiple particle states can be described by sequential two-body decays into intermediate resonances (isobars). For the $\pi^{-} \pi^{+} \pi^{-}$-states, the excited state is thus assumed to decay into a single bachelor pion and a di-pion resonance, which by itself decays again into $\pi^{+} \pi^{-}$. All known isovector and isoscalar $(\pi \pi)$ resonances have been included into the analysis. In total 42 partial waves are considered by the PWA. The waves are characterized by a set 


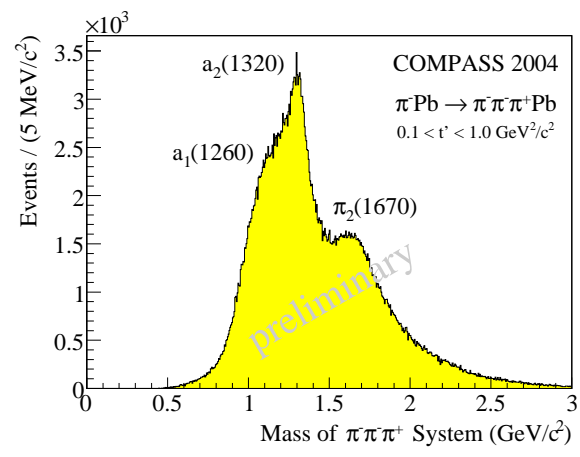

(a) invariant mass spectrum

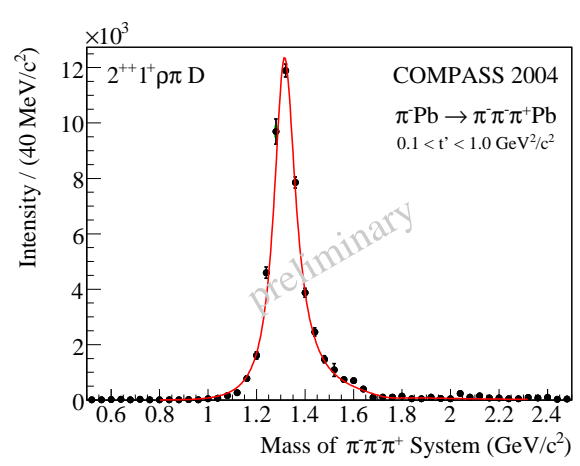

(c) $a_{2}(1320)$

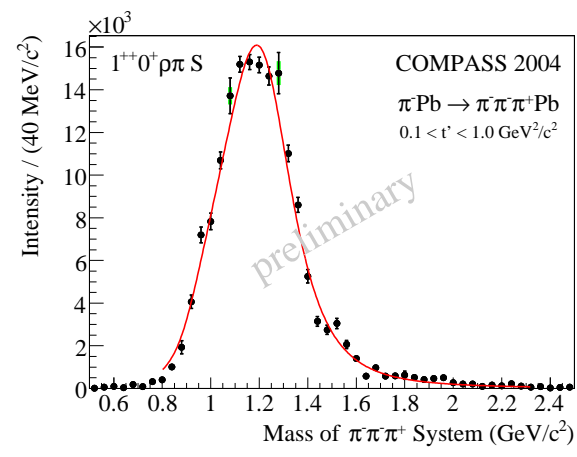

(b) $a_{1}(1260)$

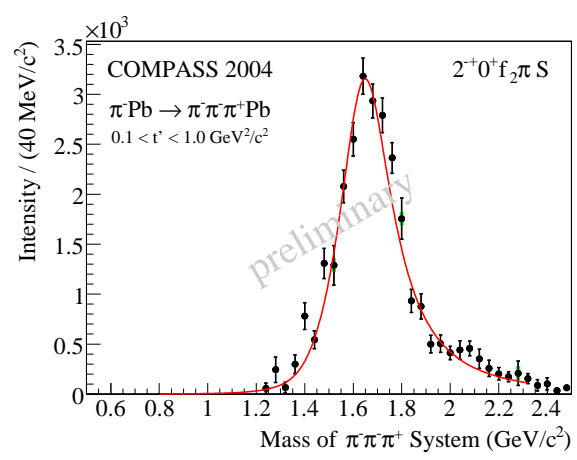

(d) $\pi_{2}(1670)$

Figure 1: a) The invariant mass spectrum of the $\pi^{-} \pi^{+} \pi^{-}$system. b)-d) Intensities of major waves $1^{++} 0^{+} \rho \pi S, 2^{++} 1^{+} \rho \pi D$, and $2^{-+} 0^{+} f_{2} \pi S$. The lines represent the result of a mass-dependent fit.

of quantum numbers $J^{P C} M^{\varepsilon}[$ isobar $] L$. Here, $M$ is the absolute value of the spin projection of the total angular momentum $J$ of the 3 pion system onto the z-axis (of the Gottfried Jackson frame); $\varepsilon$ is the reflectivity which describes the symmetry under a reflection through the production plane [R6]. $L$ is the orbital angular momentum between the isobar and the bachelor pion. For more details see reference [25].

The results obtained for the mass independent and mass dependent fit of the PWA for the three most prominent contributing waves are shown in figure $\square(\mathrm{b}-\mathrm{d})$. Figure 2(a) shows the intensity of the exotic wave $1^{-+} 1^{+} \rho \pi P$. In addition to the Breit-Wigner resonance at $1.66 \mathrm{GeV} / \mathrm{c}^{2}$, which is represented by the line to the right and can be interpreted as the $\pi_{1}(1600)$, the $1^{-+}$wave has a shoulder at lower masses (left line). This shoulder is described in our PWA by a non-resonant background, possibly caused by a Deck-like effect [27]. The resonant nature of the $1^{-+} 1^{+} \rho \pi P$ wave can be reconfirmed by the motion of its phase relative to other states. Figure [2(c) shows the phase difference between $2^{-+} 0^{+} f_{2}(980) \pi S$ and the exotic wave. No significant change is observed which can be attributed to the fact that both resonances $\pi_{1}(1600)$ and $\pi_{2}(1670)$ have similar masses and widths, causing the relative phase to be almost constant. In contrary as shown by figure 2(b), the phase difference relative to the $1^{++} 0^{+} \rho \pi S$ state clearly shows variations around $1.6 \mathrm{GeV} / \mathrm{c}^{2}$. Since the $a_{1}$ (1260) is no longer resonating at this mass, both observations can be regarded as an independent verification of the resonant origin of the $\pi_{1}(1600)$. Nevertheless it is important to note that a reconfirmation of the existence of that resonance by different final states is essential. 


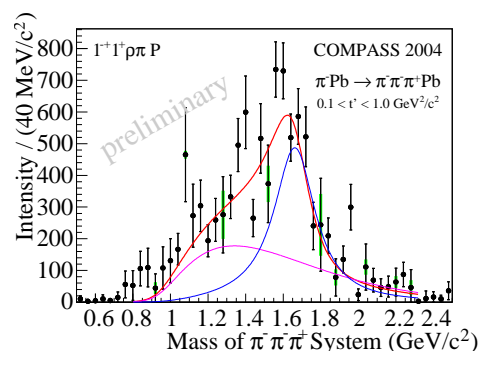

(a)

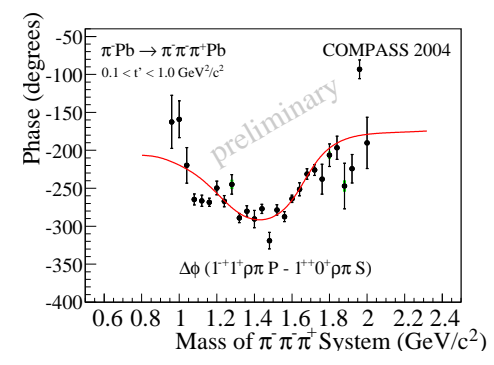

(b)

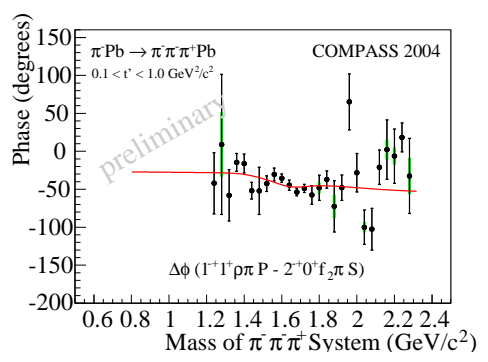

(c)

Figure 2: a) The intensity of the exotic wave $1^{-+} 1^{+} \rho \pi P$ as a function of the invariant mass. b) Phase difference between the $1^{-+} 1^{+} \rho \pi P$ and the $1^{++} 0^{+} \rho \pi S$ wave. c) Phase difference between the $1^{-+} 1^{+} \rho \pi P$ and the $2^{-+} 0^{+} f_{2} \pi S$ wave.

In this context, final states of high interest are $\rho^{-} \pi^{0}, \eta \pi^{-}, \eta^{\prime} \pi^{-}, f_{1}(1285) \pi$ and $b_{1}(1235) \pi$. The observation of a $\pi_{1}(1600)$ was already claimed by other experiments in $\eta^{\prime} \pi^{-}, f_{1}(1285) \pi$ and $b_{1}(1235) \pi$, but needs still confirmation [28].

\section{Present Status of the Analysis of 2008 and 2009 Data and Outlook}

In 2008 and 2009 COMPASS continued data taking for hadron spectroscopy at $190 \mathrm{GeV} / \mathrm{c}$ beam momentum with $\pi^{-}, K^{-}, p, \pi^{+}$beams on $\mathrm{LH}_{2}, \mathrm{~Pb}$ and $\mathrm{Ni}$ targets. The integrated luminosity was increased by two orders of magnitude compared to 2004. A number of new detectors have been installed in order to optimize the setup. This includes two CEDAR detectors in the beam line to identify beam pions or Kaons via Cherenkov radiation, a recoil proton detector consisting of two concentric rings of scintillators surrounding a $40 \mathrm{~cm}$ long liquid hydrogen target, a set of silicon microstrip detectors cooled to cryogenic temperatures for improved radiation hardness and five novel GEM detectors with pixel readout for very small area tracking. Electromagnetic calorimetry was completed and available for the first time for spectroscopy of final states with neutral particles.

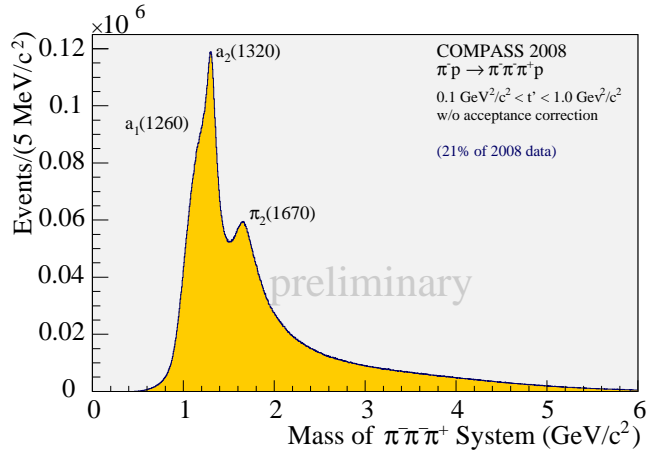

(a) $\pi^{-} p \rightarrow \pi^{-} \pi^{+} \pi^{-}$

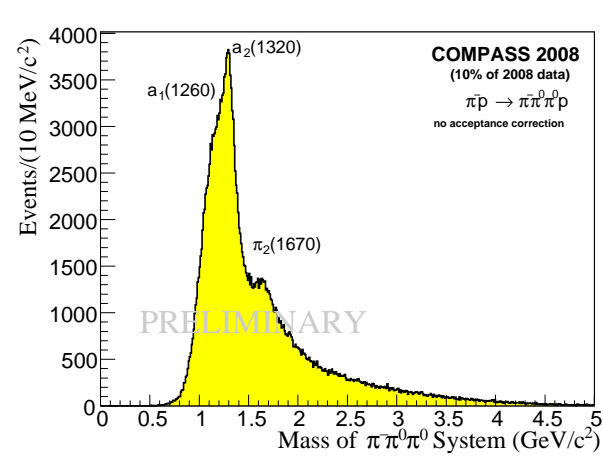

(b) $\pi^{-} p \rightarrow \pi^{-} \pi^{0} \pi^{0}$

Figure 3: Invariant mass spectra for $\pi^{-} p \rightarrow \pi^{-} \pi^{+} \pi^{-} p$ and $\pi^{-} p \rightarrow \pi^{-} \pi^{0} \pi^{0} p$ obtained in 2008. Both final state are dominated by the same major resonances $a_{1}(1260), a_{2}(1320)$ and $\pi_{2}(1670)$.

While the data analysis is presently still ongoing, a few preliminary results without acceptance corrections are released for 2008. The purpose of the following plots is to illustrate the quality 


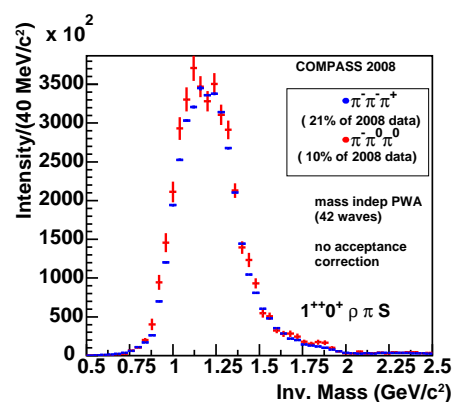

(a) $a_{1}(1260)$

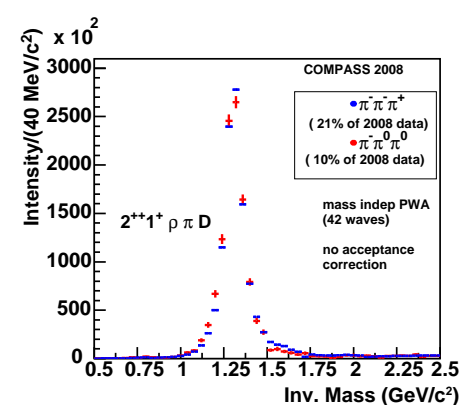

(b) $a_{2}(1320)$

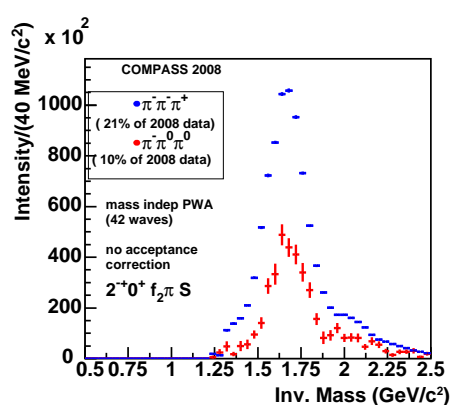

(c) $\pi_{2}(1670)$

Figure 4: a)-c) Intensities of major waves $1^{++} 0^{+} \rho \pi S, 2^{++} 1^{+} \rho \pi D$, and $2^{-+} 0^{+} f_{2} \pi S$ from the reactions $\pi^{-} p \rightarrow \pi^{-} \pi^{+} \pi^{-} p$ (blue) and $\pi^{-} p \rightarrow \pi^{-} \pi^{0} \pi^{0} p$ (red). The data are normalized for comparison to $1^{++} 0^{+} \rho \pi S$.

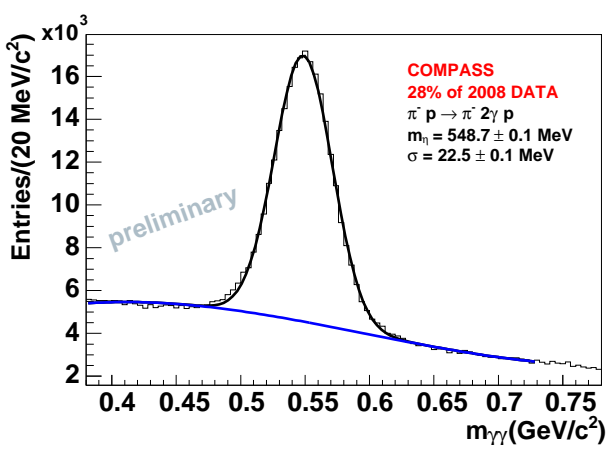

(a) $\gamma \gamma$

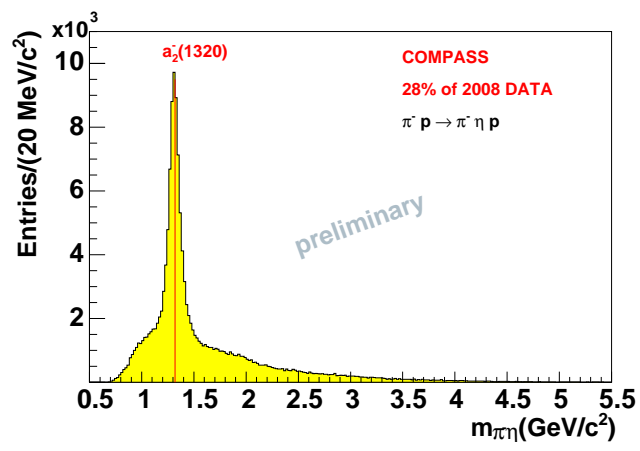

(b) $\pi^{-} \eta$

Figure 5: a) Invariant mass distribution of the $\gamma \gamma$ system for the exclusive reaction $\pi^{-} p \rightarrow \pi^{-} \gamma \gamma p$. b) Invariant mass distribution of $\pi^{-} \eta$ from $\pi^{-} p \rightarrow \pi^{-} \eta p$ with $\eta$ decaying into $\gamma \gamma$.

of the data for both charged and neutral channels for the new setup. Figure $3(\mathrm{a})$ and $3(\mathrm{~b})$ shows the invariant mass spectra for the reactions $\pi^{-} p \rightarrow \pi^{-} \pi^{+} \pi^{-} p$ and $\pi^{-} p \rightarrow \pi^{-} \pi^{0} \pi^{0} p$, respectively. Roughly 100 million events in the charged final state were collected which corresponds to 5.8 million $a_{2}(1320)$ candidates. The increase in statistics is about a factor hundred compared to 2004, as expected. For the final state $\pi^{-} \pi^{0} \pi^{0}$ roughly 10 million events are expected. The smaller reconstruction efficiency of the neutral channel can be explained in first order by the photon absorption in the spectrometer and the presently not yet optimized E-Cal reconstruction code. Both distributions look very similar and are dominated by the same major partial waves $1^{++} 0^{+} \rho \pi S, 2^{++} 1^{+} \rho \pi D$, and $2^{-+} 0^{+} f_{2} \pi S$. The intensities of these waves as a function of the invariant mass are shown for both cases in figure 1 . Again, the data were not yet acceptance corrected, but reflect the main properties as seen already by the complete analysis of 2004 data. The relative intensities of the three dominant waves of $\pi^{-} \pi^{+} \pi^{-}$and $\pi^{-} \pi^{0} \pi^{0}$ behave as expected from isospin symmetry. New precision results regarding the $\pi_{1}(1600)$ can be expected from both channels for early 2011, after a new Monte Carlo description of the setup and the calibration of the electromagnetic calorimeters will have been finalized. The PWA analysis will have to be tuned taking into account the much improved precision of the new data. 
As already mentioned in chapter 3 , the investigation of the final states $\eta \pi^{-}, \eta^{\prime} \pi^{-}, f_{1}(1285) \pi$ and $b_{1}(1235) \pi$ with high quality data are of primary importance for an unambiguous proof of a $1^{-+}$hybrid. COMPASS sees very clean signals for exclusive final states with $\eta, \eta^{\prime}$ and $f_{1}(1285)$ as the isobar and a $\pi^{-}$as the bachelor particle. The $b_{1}(1235)$ is clearly seen. For illustration, the invariant masses of the $\gamma \gamma$ and $\pi^{-} \eta$ systems of the reaction $\pi^{-} p \rightarrow \pi^{-} \eta p$ are shown in figure $\square$. Again, acceptance corrections, final E-Cal calibrations and the PWA have to be applied before new results can be presented.

\section{References}

[1] COMPASS Collaboration, E.S. Ageev, et al., Phys. Lett. B 612 (2005) 154.

[2] COMPASS Collaboration, V.Yu. Alexakhin, et al., Phys. Lett. B 647 (2007) 8.

[3] COMPASS Collaboration, V.Yu. Alexakhin, et al., Phys. Lett. B 647 (2007) 330.

[4] COMPASS Collaboration, E.S. Ageev, et al., Phys. Lett. B 633 (2006) 25.

[5] COMPASS Collaboration, M. Alekseev, et al., Phys. Lett. B 676 (2009) 31.

[6] COMPASS Collaboration, M. Alekseev, et al., Eur. Phys. J. C 52, 255-265 (2007).

[7] COMPASS Collaboration, V.Yu. Alexakhin, et al., Phys. Rev. Lett. 94, 202002 (2005).

[8] COMPASS Collaboration, E.S. Ageev, et al., Nucl. Phys. B 765 (2007) 31-70.

[9] COMPASS Collaboration, M. Alekseev, et al., Phys. Lett. B 673 (2009) 127-135.

[10] C. J.Morningstar, M. Peardon, Phys. Rev. D 60 (1999) 34509.

[11] C. Amsler and N.A. Tornqvist, Phys. Reports 389, 61 (2004).

[12] K. J. Juge, J. Kuti, and C. Morningstar, AIP Conf. Proc. 688, 193 (2004).

[13] D. R. Thompson et al., Phys. Rev. Lett. 79, 1630 (1997).

[14] V. Dorofeev et al., AIP Conf. Proc. 619, 143 (2002).

[15] A. Abele et al., Phys. Lett. B423, 175 (1998).

[16] A. Abele et al., Phys. Lett. B446, 349 (1999).

[17] G. S. Adams et al., Phys. Rev. Lett. 81, 5760 (1998).

[18] S. U. Chung et al., Phys. Rev. D65, 072001 (2002).

[19] Y. Khokhlov, Nucl. Phys. A663, 596 (2000).

[20] G. M. Beladidze et al., Phys. Lett. B313, 276 (1993).

[21] E. I. Ivanov et al., Phys. Rev. Lett. 86, 3977 (2001).

[22] J. Kuhn et al., Phys. Lett. B595, 109 (2004).

[23] D. V. Amelin et al., Phys. Atom. Nucl. 68, 359 (2005).

[24] M. Lu et al., Phys. Rev. Lett. 94, 032002 (2005).

[25] COMPASS Collaboration, V.Yu. Alexakhin, et al., Phys. Rev. Lett. 104, 241803 (2010).

[26] S. U. Chung and T. L. Trueman, Phys. Rev. D11, 633 (1975).

[27] R. T. Deck, Phys. Rev. Lett. 13, 169 (1964).

[28] K. Nakamura et al. (Particle Data Group), JPG 37, 075021 (2010). 